\title{
Advice to Bottle-feeding Parents could Defer Obesity
}

\section{Pauline Emmett}

Centre for Child and Adolescent Health Population Health Sciences, Bristol Medical School, University of Bristol, United Kingdom

*Corresponding author: Pauline Emmett, Centre for Child and Adolescent Health Population Health Sciences, Bristol Medical School, University of Bristol, United Kingdom, Tel: (0117)3310195; E-mail: p.m.emmett@bristol.ac.uk

Rec date: Sep 04, 2017; Acc date: Oct 7, 2017; Pub date: Oct 13, 2017

Copyright: (C) 2017 Emmett PM. This is an open-access article distributed under the terms of the Creative Commons Attribution License, which permits unrestricted use, distribution, and reproduction in any medium, provided the original author and source are credited.

Citation: Emmett PM (2017) Advice to bottle-feeding parents could defer obesity. J Child Obes Vol No 2 Iss No: 3:14.

\section{Letter to Editor}

Fast early growth may be related to the process of bottle feeding rather than solely the type of milk fed. Bottle-feeding parents should be advised on the amount of milk to feed and how to feed their child responsively.

The seeds of obesity are often sown in infancy and fast early growth is associated with obesity in childhood. There is conflicting evidence about whether being breastfed protects children against obesity. Formula milk has been designed to mimic breastmilk as far as possible chemically but it cannot mimic the mode of delivery of breastmilk. Perhaps this important difference is one of its problems.

Many studies have compared breast and formula-fed infants but only a few have assessed the actual amounts of breast, formula or other milks fed or the contribution to energy intake of complementary foods and drinks and very few have investigated the effect of using a bottle for milk-feeding.

One study which has investigated infant feeding in detail is the Avon Longitudinal Study of Parents and Children (ALSPAC) in the UK where dietary diary data was recorded by parents at 4,8 and 18 months in around 1000 children and their growth followed up to 10 years of age. The nutrition group lead by Pauline Emmett has investigated the contribution of different milks and complementary foods to energy intake and growth [1, 2]. When diet was assessed at 4 months of age higher energy intake predicted greater weight gain between birth and age 1,2 and 3 years and larger body weight and BMI at 5 years of age in formula or mixed-fed children but there was no association in breastfed children [1]. Assessing diet at 8 months showed that where infants were feed more than the recommended amount ( $600 \mathrm{~mL}$ or 21 ounces/day) of formula (or cows' milk) they grew faster in the first 2 years, and beyond in the case of cows' milk, both in weight and height than breastfed infants [2]. The dietary data revealed that the energy contribution from foods and drinks other than milk was lower in both formula and cow's milk groups fed more than $600 \mathrm{~mL}$ of milk than in their counterparts fed less than $600 \mathrm{~mL}$ of milk, however, this did not compensate fully for the extra energy obtained from the milk feeds. These data suggest that dysregulation of intake and/or over feeding may be a problem in bottle-fed infants.
The Infant Feeding Practices Study II in USA, a longitudinal study of around 2000 new mothers and their infants, collected information by questionnaire about milk feeding and current weight 10 times during the first year of the infant's life. Details of the frequency and mode of delivery of feeds and the types of milk fed were collected [3]. Although $83 \%$ of mothers' breast fed initially only $50 \%$ still did so when their infant was 6 months old and in the second 6 months of life up to $70 \%$ of infants were fed formula [3]. At 10 months of age $40 \%$ of formula drinkers were taking 7 ounces or more per feed and an average of 4 feeds per day (possibly up to 28 ounces/day).

The authors led by Sara Fein and Lawrence Grummer-Strawn carefully investigated the differences in weight gain associated with the mode of delivery of the milk and found that compared with infants fed only at the breast, both those fed formula only or breast milk by bottle gained more weight per month [4]. In infants fed only breast milk weight gain increased as numbers of feedings by bottle increased. A further detailed analysis looked at bottle emptying by infants. This showed that for infants who were exclusively fed from the breast in early infancy $27 \%$ emptied the bottle in late infancy where as $54 \%$ of infants fed both breast and bottle and $68 \%$ of infants fed only by bottle did so [5]. The intensity of early breastfeeding was also important; if over $80 \%$ of feeds were from the breast the infant was less likely to have excess weight gain in the second 6 months of life [6]. There was an independent relationship between infants who often emptied the bottle in early infancy and risk of excess weight gain which was not mediated by parental encouragement [6]. It seems that bottle-feeding may have a distinct effect on infants' dysregulation of milk intake compared with feeding from the breast.

Alison Ventura followed up on this work with an experimental approach in 21 formula-feeding mother and infant pairs [7]. In one group the mothers continued with their usual feeding practices and in the other group mothers were helped to be very responsive to infant cues of hunger and satiety. Mothers also completed a questionnaire about their feeding styles and infant's temperament. Infants in the mother-led group consumed around $42 \%$ more formula than infants in the infantled group. Greater intakes in the mother-led group were predicted by both feeding style (less restriction and less responsiveness) and infant temperament (more positive mood). A longitudinal study of growth in twins, the UK Gemini study 
started by Jane Wardle, also looked at maternal feeding styles in relation to bottle-feeding [8]. The general appetite of the child was rated along with the use of restrictive feeding practices. Of the 1920 twin infants studied 70\% were bottle-fed at 3 months. In bottle-fed but not breastfed infants there was an association between large appetite and more use of restriction by the mother (Odds Ratio=1.52, 95\% Confidence Interval: 1.13-2.04, $\mathrm{p}=0.006$ ). It is likely therefore that both maternal and child factors are important in determining the amount of milk ingested by bottle-fed infants.

In addition to the necessary support for breastfeeding parents, bottle-feeding parents should be advised not to encourage their infant to finish milk offered in a bottle/cup, to gradually decrease the amount of milk offered at each bottle/cup feed as complementary feeding is established and to be responsive to their child's cues of hunger and satiety. The UK recommendation of not more than $600 \mathrm{~mL}$ (21 ounces) of milk per day in late infancy seems sensible.

\section{References}

1. Ong KK, Emmett PM, Noble S, Ness A, Dunger DB, et al. (2006) Dietary energy intake at the age of 4 months predicts postnatal weight gain and childhood body mass index. Pediatrics 117: e503-08.
2. Hopkins D, Steer CD, Northstone K, Emmett PM (2015) Effects on childhood body habitus of feeding large volumes of cow or formula milk compared with breastfeeding in the latter part of infancy. Am J Clin Nutr 102: 1096-1103.

3. Grummer-Strawn LM, Scanlon KS, Fein SB (2008) Infant feeding and feeding transitions during the first year of life. Pediatrics 122 Suppl 2: S36-42.

4. Li R, Magadia J, Fein SB, Grummer-Strawn LM (2012) Risk of bottle-feeding for rapid weight gain during the first year of life. Arch Pediatr Adolesc Med 166(5):431-6.

5. Li R, Fein SB, Grummer-Strawn LM (2010) Do infants fed from bottles lack self-regulation of milk intake compared with directly breastfed infants? Pediatrics 125: e1386-93.

6. Li R, Fein SB, Grummer-Strawn LM (2008) Association of breastfeeding intensity and bottle-emptying behaviors at early infancy with infants' risk for excess weight at late infancy. Pediatrics 122: S77-84.

7. Ventura AK, Mennella JA (2017) An Experimental Approach to Study Individual Differences in Infants' Intake and Satiation Behaviors during Bottle-Feeding. J Child obes 13: 44-52.

8. Fildes A, van Jaarsveld CH, Llewellyn C, Wardle J, Fisher A (2015) Parental control over feeding in infancy. Influence of infant weight, appetite and feeding method. Appetite 91: 101-16. 